\title{
A Two Species Amensalism Model with Harvesting By Homotopy Analysis Method
}

\author{
B. Sita Rambabu ${ }^{1}$, K.L.Narayan ${ }^{2}$, Sahanaz Bathul ${ }^{3}$ \\ 1. Department of Mathematics, Vidyajyothi Institute of Technology, Hyderabab \\ 2. Department of Mathematics, Vignan Institute of Technology \& Science, Hyderabad \\ 3. Department of Mathematics, JNT University, Kukatpally, Hyderabad \\ Email Id: sitarambabu.b@gmail.com
}

Keywords: Amensalism, series solution, zero order deformation, embedded parameter, linear operator and HAM.

\begin{abstract}
In this paper a two species Amensalism model is taken up for analytical study. The model comprises an Amensal, which is harvested at a rate proportional to their population and an enemy. Moreover both the species are provided with limited resources. The series solution of the non-linear system was approximated by the Homotopy analysis method (HAM) and the results are supported by numerical simulations.
\end{abstract}

\section{Introduction:}

Symbioses are a broad class of interactions among organisms --Amensalism involves one organism affecting another negatively without any positive or negative benefit for itself e.g., avian botulism and "red tides" (caused by dino flagellates) are often extremely toxic for birds, marine mammals, humans, etc.

Amensalism may lead to the pre-emptive colonization of a habitat. Once an organism establishes Itself within a habitat it may prevent other populations from surviving in that habitat. The production of lactic acid or similar low-molecular-weight fatty acids is inhibitory to many bacterial populations. Populations able to produce and tolerate high concentrations of lactic acids, for example, are able to modify the habitat so as to preclude the growth of other bacterial populations. E. coli is unable to grow in the rumen, probably because of the presence of volatile fatty acids produced there by anaerobic heterotrophic microbial populations. Fatty acids produced by microorganisms on skin surfaces are believed to prevent the colonization of these habitats by other microorganisms. Populations of yeasts on skin surfaces are maintained in low numbers by microbial populations producing fatty acids. Acids produced by microbial populations in the vaginal tract are probably responsible for preventing infection by pathogens such as Candida albicans

In the present investigation a two species Amensalism model with harvesting with limited resources for both the species was taken up for analytic study. The model is represented by coupled non-linear ordinary differential equations. The series solution of the non-linear system is approximated by Homotopy Analysis Method.

The governing equations of the system are as follows

$$
\begin{aligned}
& \frac{d x}{d t}=a_{1}(1-k) x(t)-\alpha_{11} x^{2}(t)-\alpha_{12} x(t) y(t) \\
& \frac{d y}{d t}=a_{2} y(t)-\alpha_{22} y^{2}(t)
\end{aligned}
$$

With the notation

$\mathrm{x}(\mathrm{t}), \mathrm{y}(\mathrm{t})$ : populations of the species1 and species2

$\mathrm{a}_{1}, \mathrm{a}_{2}$ : rates of natural growth of species 1 and species 2 .

$\alpha_{11}, \alpha_{22}$ : rates of decrease due to insufficient food of the species 1 and species 2 . 
$\alpha_{12}$ : rate of decrease of the species 1 due to inhibition by the species 2 .

$\mathrm{K}$ : rate of harvesting proportional to the population size of species $1(0<\mathrm{k}<1)$

\section{Basic ideas of HAM:}

In this paper, we apply the homotopy analysis method to the discussed problem. To show the basic idea, let us consider the following differential equation

$$
\mathrm{N}(\mathrm{u}, \mathrm{t})=0 \text {, }
$$

Where $\mathrm{N}$ is a nonlinear operator, $\mathrm{t}$ denote independent variable, $\mathrm{u}(\mathrm{t})$ is an unknown function, respectively. For simplicity, we ignore all boundary or initial conditions, which can be treated in the similar way. By means of generalizing the traditional homotopy method, Liao constructs the socalled zero-order deformation equation

$$
(1-p) L\left[\phi(t ; p)-u_{0}(t)\right]=p \bar{h} H(t) N[\phi(t ; p)]
$$

Where $p \in[0,1]$ is the embedding parameter, $\bar{h}$ is a nonzero auxiliary, parameter $\mathrm{H}$ is an auxiliary, parameter $\mathrm{H}$ is an auxiliary function $\mathrm{L}$ is an auxiliary linear operator, $\mathrm{u}_{0}(\mathrm{t})$ is an initial guess of $\mathrm{u}(\mathrm{t}), \varphi(\mathrm{t}, \mathrm{p})$ is a unknown function, respectively. It is important that one has great freedom to choose auxiliary things in HAM. Obviously, When

$$
\mathrm{P}=0 \text { and } \mathrm{p}=1 \text {, it holds } \varphi(\mathrm{t}, 0)=\mathrm{u}_{0}(\mathrm{t}), \varphi(\mathrm{t}, 1)=\mathrm{u}(\mathrm{t})
$$

Respectively. Thus as $\mathrm{p}$ increases from 0 to 1 , the solution $\varphi(t ; p)$ varies from the initial guesses $\mathrm{u}_{0}(\mathrm{t})$ to the solution $\mathrm{u}(\mathrm{t})$. Expanding $\varphi(t ; p)$ in Taylor series with respect to $\mathrm{p}$, one has

$$
\phi(t ; p)=u_{0}(t)+\sum_{m=1}^{+\infty} u_{m}(t) p^{m}
$$

Where

$$
u_{m}(t)=\left.\frac{1}{m !} \frac{\partial^{m} \phi(t ; p)}{\partial p^{m}}\right|_{p=0}
$$

If the initial guess (2.3), the auxiliary linear parameter $\mathrm{L}_{\mathrm{i}}$, the non-zero auxiliary parameter $\mathrm{h}_{\mathrm{i}}$ and the auxiliary function $\mathrm{H}_{\mathrm{i}}$ are properly choosen, so that the power series (2.4) converges at $\mathrm{p}=1$.

Then we have under these assumptions the solution series

$$
u(t)=u_{0}(t)+\sum_{m=1}^{+\infty} u_{m}(t)
$$

Which must be one of solution s of original non linear equation, as proved by Liao [15].As $\bar{h}=-1$ and $\mathrm{H}(\mathrm{t})=1, \mathrm{Eq}(2.2)$ becomes $(1-p) L\left[\phi(t ; p)-u_{0}(t)\right]+p N[\phi(t ; p)]=0$,

which is used mostly in the homotopy perturbation method, whereas the solution obtained directly, without using Taylor series which is explained by H.Jafari,M.Zabihi and M.Saidy [14] and J.H.He $[15,16]$ and S.J.Liao [17] compare the HAM and HPM. According to the definition, the governing equation can be deduced from the zero-order deformation equation (2.7) . 
Define the vector $\vec{u}_{k}=\left\{u_{0}, u_{1}, \ldots . . u_{k}\right\} \quad$ Differentiating Eq. (2.7), $\mathrm{m}$ times with respect to embedding parameter $\mathrm{p}$ and then setting $\mathrm{p}=0$ and finally dividing them by $\mathrm{m}$ !, we have the so-called mth-order deformation equation

$$
\begin{aligned}
L\left[u_{m}(t)-\chi_{m} u_{m-1}(t)\right] & =\bar{h} H(t) R_{m}\left(\vec{u}_{m-1}\right), \\
R_{m}\left(\vec{u}_{m-1}\right) & =\left.\frac{1}{(m-1) !} \frac{\partial^{m-1} N[\phi(t ; p)]}{\partial p^{m-1}}\right|_{p=0},
\end{aligned}
$$

Where

and

$$
\chi_{m}=\left\{\begin{array}{l}
0, m \leq 1, \\
1, m>1 .
\end{array}\right.
$$

\section{Application:}

Consider the nonlinear differential equation(1.1) with initial conditions .we assume the solution of the system $(1.1), x(t), y(t)$ can be expressed by following set of base functions in the form

$$
x(t)=\sum_{m=1}^{+\infty} a_{m} t^{m}, y(t)=\sum_{m=1}^{+\infty} b_{m} t^{m}
$$

Where $a_{m}, b_{m}$ are coefficients to be determined. This provides us the so called rule of solution expression i.e., the solution of (1.1) must be expressed in the same from as (3.1) and the other expressions must be avoided. According to (1.1) and (3.1) we chose the linear operator. To solve the system of Eqs.(1.1),Homotopy analysis method is employed. We consider the following initial approximations

$$
x_{0}(t)=x(t=0)=x_{0} \quad y_{0}(t)=y(t=0)=y_{0}
$$

The linear and non-linear operators are denoted as follows.

$$
\begin{aligned}
& L_{1}[x(t ; p)]=\frac{d x(t ; p)}{d t}, L_{2}[y(t ; p)]=\frac{d y(t ; p)}{d t} \\
& N_{1}[x(t, p)]=\frac{d x(t ; p)}{d t}-a_{1}(1-k) x(t ; p)+\alpha_{11} x^{2}(t ; p)+\alpha_{12} x(t ; p) y(t ; p) \\
& N_{2}[y(t, p)]=\frac{d y(t ; p)}{d t}-a_{2} y(t ; p)+\alpha_{22} y^{2}(t ; p)
\end{aligned}
$$

Using above definition the zero order deformation equation can be constructed

$$
\begin{aligned}
& (1-p) L_{1}\left[x(t ; p)-x_{0}(t)\right]=p h_{1} N_{1}[x, y], \\
& (1-p) L_{2}\left[y(t ; p)-y_{0}(t)\right]=p h_{2} N_{2}[x, y],
\end{aligned}
$$

When $\mathrm{p}=0$ and $\mathrm{p}=1$,from the zero-deformation equations one has,

$$
\begin{aligned}
& x(t ; 0)=x_{0}(t) \quad x(t ; 1)=x(t) \\
& y(t ; 0)=y_{0}(t) \quad y(t ; 1)=y(t)
\end{aligned}
$$


And expanding $x(t ; p)$ and $y(t ; p)$ in Taylors series, with respect to embedding parameter $p$. one obtains

$$
\begin{aligned}
x(t ; p) & =x_{0}(t)+\sum_{m=1}^{+\infty} x_{m}(t) p^{m} \\
y(t ; p) & =y_{0}(t)+\sum_{m=1}^{+\infty} y_{m}(t) p^{m} \\
x_{m}(t) & =\left.\frac{1}{m !} \frac{d^{m} x(t ; p)}{d p^{m}}\right|_{p=0} \\
y_{m}(t) & =\left.\frac{1}{m !} \frac{d^{m} y(t ; p)}{d p^{m}}\right|_{p=0} \\
p=1 & \left\{\begin{array}{l}
x_{m}(t)=x_{0}(t)+\sum_{m=1}^{+\infty} x_{m}(t) \\
y_{m}(t)=y_{0}(t)+\sum_{m=1}^{+\infty} y_{m}(t)
\end{array}\right.
\end{aligned}
$$

Define the vector

$$
\begin{aligned}
& \vec{x}_{m}=\left[x_{0}(t), x_{1}(t), \ldots . x_{m}(t)\right] \\
& \vec{y}_{m}=\left[y_{0}(t), y_{1}(t), \ldots . y_{m}(t)\right]
\end{aligned}
$$

And apply the procedure stated before. The following $\mathrm{m}^{\text {th }}$-order deformation Eq will be achieved.

$$
\begin{aligned}
& L_{1}\left[x_{m}(t)-\chi_{m} x_{m-1}(t)\right]=\bar{h}_{1} H_{1}(t) R_{1 m}\left(\vec{x}_{m-1}, \vec{y}_{m-1}\right), \\
& L_{2}\left[y_{m}(t)-\chi_{m} y_{m-1}(t)\right]=\bar{h}_{2} H_{2}(t) R_{2 m}\left(\vec{x}_{m-1}, \vec{y}_{m-1}\right),
\end{aligned}
$$

Let us consider $H_{1}(t)=H_{2}(t)=1$ and the initial conditions

$$
\begin{aligned}
& x_{0}(t)=x(t=0)=x_{0} \quad y_{0}(t)=y(t=0)=y_{0} \text { in above equations } \\
& R_{1 m}\left(x_{m-1}, y_{m-1}\right)=\frac{1}{(m-1) !} \frac{d^{m-1}}{d p^{m-1}} N[x(t, p)]=\frac{d}{d t} x_{m-1}(t)-a_{1}(1-k) x_{m-1}+\alpha_{11} \sum_{n=1}^{m} x_{n}(t) x_{m-n-1}(t)+\alpha_{12} \sum_{n=0}^{m-1} x_{n}(t) y_{m-n-1}(t) \\
& R_{2 m}\left(x_{m-1}, y_{m-1}\right)=\frac{1}{(m-1) !} \frac{d^{m-1}}{d p^{m-1}} N[y(t, p)]=\frac{d}{d t} y_{m-1}(t)-a_{2} y_{m-1}+\alpha_{22} \sum_{n=1}^{m} y_{n}(t) y_{m-n-1}(t)
\end{aligned}
$$

The following will be obtained successively

$$
\begin{aligned}
& L_{1}\left(x_{1}(t)-\chi_{1} x_{0}(t)\right)=h_{1}\left[\frac{d}{d t} x_{0}(t)-a_{1}(1-k) x_{0}(t)+\alpha_{11} x_{0}^{2}(t)+\alpha_{12} x_{0}(t) y_{0}(t)\right] \\
& \chi_{m}(t)=0, m \leq 0 \\
& \quad 1, m>0 \\
& L_{1}\left(x_{1}(t)\right)=h_{1}\left[-a_{1}(1-k) x_{0}(t)+\alpha_{11} x_{0}^{2}(t)+\alpha_{12} x_{0}(t) y_{0}(t)\right] \\
& x_{1}(t)=h_{1}\left[-a_{1}(1-k) x_{0}+\alpha_{11} x_{0}^{2}+\alpha_{12} x_{0} y_{0}\right] t \\
& L_{1}\left(y_{1}(t)-\chi_{1} y_{0}(t)\right)=h_{2}\left[\frac{d}{d t} y_{0}(t)-a_{2} y_{0}(t)+\alpha_{22} y_{0}^{2}(t)\right] \\
& L_{1}\left(y_{1}(t)\right)=h_{2}\left[-a_{2} y_{0}(t)+\alpha_{22} y_{0}^{2}(t)\right] \\
& y_{1}(t)=h_{2}\left[-a_{2} y_{0}+\alpha_{22} y_{0}^{2}\right] t
\end{aligned}
$$




$$
\begin{aligned}
& L_{1}\left(x_{2}(t)-\chi_{2} x_{1}(t)\right)=h_{1}\left[\frac{d}{d t} x_{1}(t)-a_{1}(1-k) x_{1}(t)+\alpha_{11} \sum_{n=0}^{1} x_{n}(t) x_{1-n}(t)+\alpha_{12} \sum_{n=0}^{1} x_{n}(t) y_{1-n}(t)\right] \\
& x_{2}(t)=\left(h_{1}+h_{1}^{2}\right) M_{1} t+\frac{t^{2}}{2}\left[-a_{1}(1-k) h_{1}^{2} M_{1}+2 \alpha_{11} x_{0} h_{1} M_{1}+\alpha_{12} y_{0} M_{1}+h_{2} a_{2} \alpha_{12} x_{0}\right]
\end{aligned}
$$

where $M_{1}=-a_{1}(1-k) x_{0}+\alpha_{11} x_{0}^{2}+\alpha_{12} x_{0} y_{0}$

$L_{1}\left(y_{2}(t)-\chi_{2} y_{1}(t)\right)=h_{2}\left[\frac{d}{d t} y_{1}(t)-a_{2} y_{1}(t)+\alpha_{22} \sum_{n=0}^{1} y_{n}(t) y_{1-n}(t)\right]$

$y_{2}(t)=\left(h_{2}+h_{2}^{2}\right)\left[-a_{2} y_{0}+\alpha_{22} y_{0}^{2}\right] t+\frac{h_{2}^{2} y_{0}}{2}\left[\left(a_{2}^{2}-3 a_{2} \alpha_{22} y_{0}+2 \alpha_{22}^{2} y_{0}^{2}\right)\right] t^{2}$

$L_{1}\left(x_{3}(t)-\chi_{3} x_{2}(t)\right)=h_{1}\left[\frac{d}{d t} x_{2}(t)-a_{1} x_{2}(t)+\alpha_{11} \sum_{n=0}^{2} x_{n}(t) x_{1-n}(t)+\alpha_{12} \sum_{n=0}^{2} x_{n}(t) y_{1-n}(t)\right]$

$x_{3}(t)=\left(1+h_{1}\right)\left(h_{1}+h_{1}^{2}\right) M_{1} t+\left[\left(1+h_{1}\right) M_{2}+h_{1} \alpha_{12} y_{0}\left(h_{1}+h_{1}^{2}\right) M_{1}+\alpha_{12} h_{1} x_{0}\left(h_{2}+h_{2}^{2}\right)\left(-a_{2} y_{0}+\alpha_{22} y_{0}^{2}\right)\right] \frac{t^{2}}{2}$

$+\left[-2 a_{1} h_{1} M_{2}+2 h_{1} \alpha_{11} M_{2} x_{0}+\alpha_{11} h_{1}^{3} M_{1}^{2}+2 h_{1} \alpha_{12} h_{2}{ }^{2} x_{0} y_{0}{ }^{2}\left(a_{2}{ }^{2}-3 a_{2} \alpha_{22} y_{0}+2 \alpha_{22}{ }^{2} y_{0}{ }^{2}\right)+h_{1}{ }^{2} h_{2} \alpha_{12} M_{1}\left(-a_{2} y_{0}+\alpha_{22} y_{0}{ }^{2}\right)+2 h_{1} \alpha_{12} M_{2} y_{0}\right] \frac{t^{3}}{3}$

$M_{2}=\left[-a_{1} h_{1}^{2}(1-k) M_{1}+2 h_{1} \alpha_{11} x_{0} M_{1}+\alpha_{12} y_{0} M_{1}+h_{2} \alpha_{12} \alpha_{22} x_{0} y_{0}{ }^{2}-h_{2} \alpha_{12} a_{2} x_{0}\right]$

$L_{1}\left(y_{3}(t)-\chi_{3} y_{2}(t)\right)=h_{2}\left[\frac{d}{d t} y_{2}(t)-a_{2} y_{2}(t)+\alpha_{22} \sum_{n=0}^{2} y_{n}(t) y_{1-n}(t)\right]$

$y_{3}(t)=\left(1+h_{2}\right) k_{3} t+\left[\left(1+h_{2}\right) k_{4}-a_{2} h_{2} k_{3}+2 h_{2} k_{3} \alpha_{22} y_{0}\right] \frac{t^{2}}{2}+\left[\left(-\frac{1}{2} a_{2} h_{2} k_{4}+h_{2} \alpha_{22} k_{4} y_{0}+\frac{\alpha_{22} h_{2}^{3} k_{3}^{2}}{\left(h_{2}+h_{2}^{2}\right)^{2}}\right)\right] \frac{t^{3}}{3}$

Where

$$
\begin{aligned}
& k_{1}=\left[-a_{1} x_{0}+\alpha_{11} x_{0}^{2}+\alpha_{12} x_{0} y_{0}\right] \\
& k_{2}=\frac{h_{1} x_{0}}{2}\left[h_{1}\left(-a_{1}+\alpha_{11} x_{0}+\alpha_{12} y_{0}\right)\left(-a_{1}+2 \alpha_{11} x_{0}+\alpha_{12} y_{0}\right)+h_{2} \alpha_{12}\left(-a_{2} y_{0}+\alpha_{22} y_{0}^{2}\right)\right] \\
& k_{3}=\left(h_{2}+h_{2}^{2}\right)\left[-a_{2} y_{0}+\alpha_{22} y_{0}^{2}\right] \\
& k_{4}=\frac{h_{2}^{2} y_{0}}{2}\left[\left(a_{2}^{2}-3 a_{2} \alpha_{22} y_{0}+2 \alpha_{22}^{2} y_{0}^{2}\right)\right]
\end{aligned}
$$

The three terms approximation to the solution will be considered as

$$
\begin{aligned}
& x(t) \approx x_{0}+x_{1}(t)+x_{2}(t)+x_{3}(t) \\
& y(t) \approx y_{0}+y_{1}(t)+y_{2}(t)+y_{3}(t)
\end{aligned}
$$


4. Numerical example:

$\mathrm{x} 0=5 ; \mathrm{y} 0=10 ; \mathrm{a} 1=.5 ; \mathrm{a} 2=.09 ; \mathrm{a} 11=05 ; \mathrm{a} 12=2.6 ; \mathrm{a} 22=7 ; \mathrm{h} 1=1 ; \mathrm{h} 2=1 ;$

$\mathrm{k}=.05$;

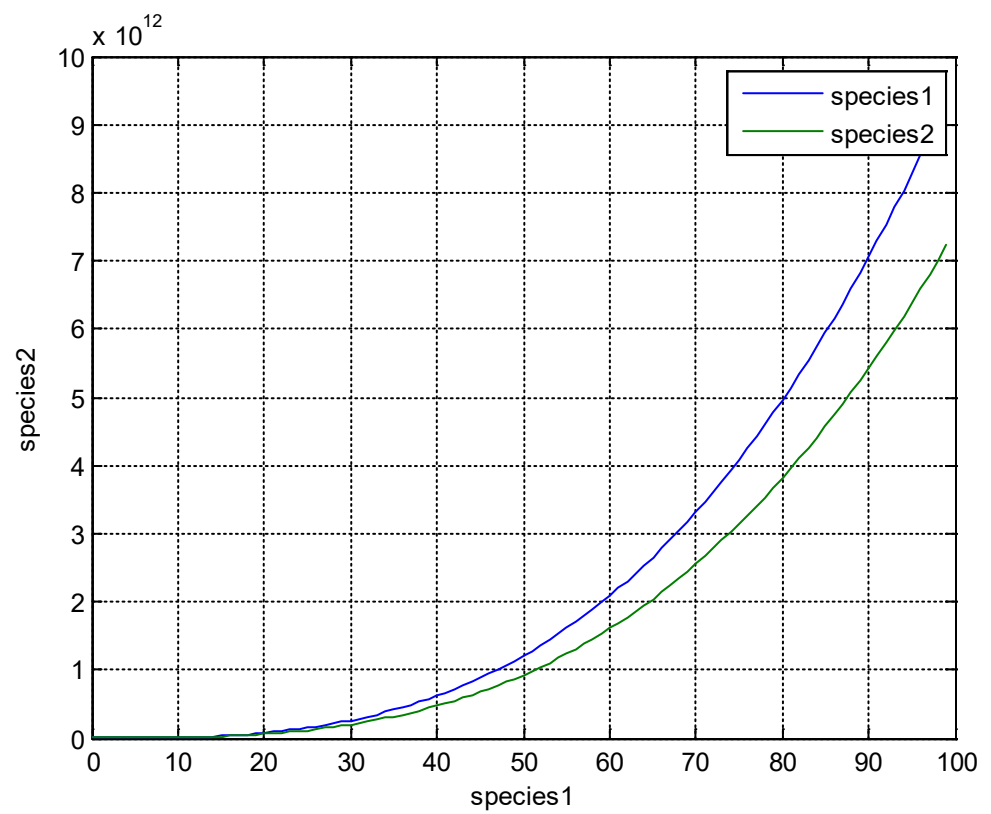

Fig 5.1: The Variation of $x$ and $y$ with respective Time (t) for system of Eq (1.1)

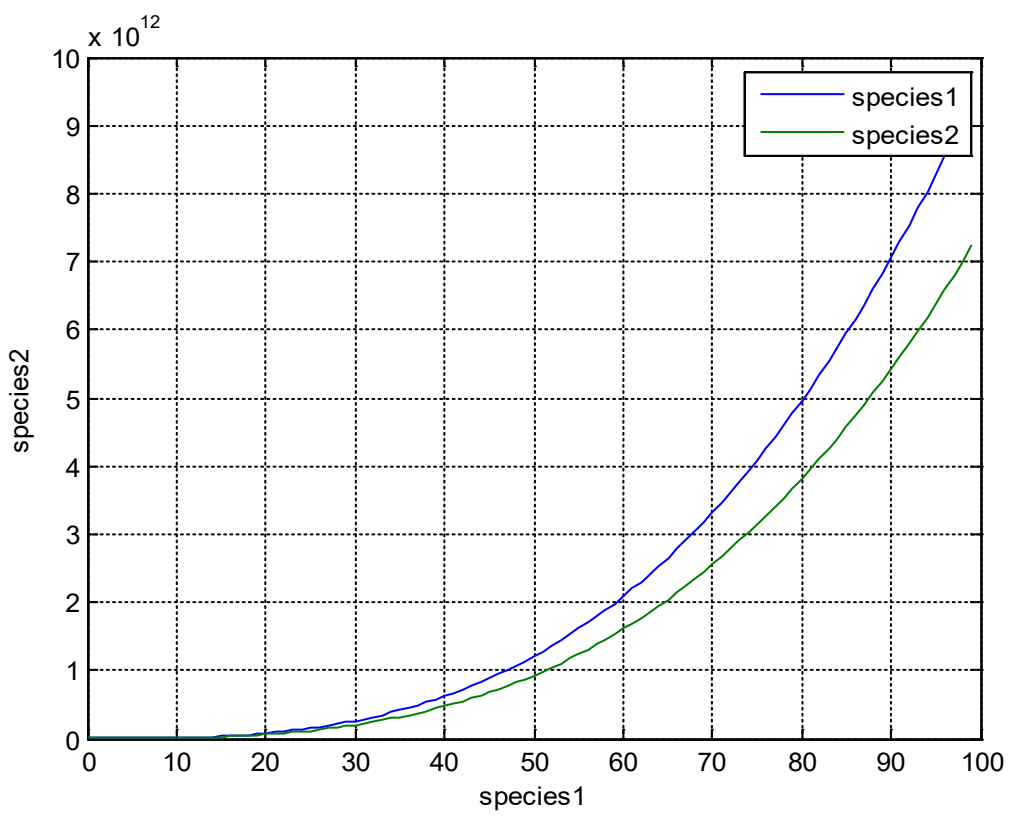

Fig 5.2: The Variation of $x$ and $y$ with respective Time (t) for system of $\mathbf{E q}(1.1)$ with $k=0$

\section{Conclusion:}

In the analysis of considered two species Amensalism model with harvesting of the species 1,we discussed the series approximations of the model by homotopy analysis method and also discussed the effect of harvesting of the species1. From the graphs shown in fig 5.1 and 5.2 it is evident that harvesting on species 1 does not have any influence on the stability. 


\section{References:}

[1]. Acharyulu K.V.L.N. \& Pattabhi Ramacharyulu. N.Ch. "An Amensal-Enemy specie pair with limited and unlimited resources respectively-A numerical approach", Int.J.Open problems Compt.Math (IJOPCM), Vol.3.No.1.pp.73-91., March2010.

[2]. Acharyulu K.V.L.N. \& Pattabhi Ramacharyulu. N.Ch.”On The stability of An AmensalHarvested Enemy Species pair with limited resources", International journal of computational Intelligence Research (IJCIR), Vol.6, No.3; pp.343-358, June 2010.

[3]. Acharyulu K.V.L.N. \& Pattabhi Ramacharyulu. N.Ch;"On the stability of an enemy -Amensal species pair with limited resources", International Journal of Applied Mathematical Analysis and Applications.vol 4,No 2,pp.149-161,July2009.

[4]. Acharyulu K.V.L.N. \& Pattabhi Ramacharyulu. N.Ch. ;’On An Amensal-Enemy Ecological Model With Variable Amensal Coefficient" is accepted for publication in "International Journal of Computational cognition(IJCC)",Yang's Scientific Research Institute, USA(in press).

[5] Shiva Prakash \& T.Karunanithi;" Modeling of interaction between Schizosaccharomyces pombe and Saccharomyces cerevisiae to predict stable operating conditions in a chemo stat (IJCRGG)", International Journal of Chem. Tech Research Coden (USA).

[6] S.J.Liao,The proposed homotopy analysis technique for the solution of nonlinear problems,PhD thesis,Shanghai Jiao Tong University 1992.

[7] S.J.Liao Beyond perturbation: introduction to the homotopy analysis method.CRC Press, Boca Raton: Chapman \& Hall (2003)

[8] S.J.Liao. On the homotopy analysis method for nonlinear problems. Appl.Math. Compat., 147(2004), 499-513.

[9] S.J.Liao. Comparison between the homotopy analysis method and homotopy perturbation method. Appl Math Comput.,169(2005),1186-1194.

[10] S.J.Liao A new branch of solutions of boundary-layer flows over an impermeable stretched plate. Int J Heat Mass Transfer.,.48(2005),2529-2539.

[11] M.Ayub, A.Rasheed, T.Hayat.Exact flow of a third grade fluid past a porous plate using homotopy analysis method.Int J Eng Sci..41(2003), 2091-2103.

[12] T.Hayat, M.Khan. Homotopy solutions for a generalized second-grade fluid past a porous plate. Nonlinear Dyn.,42:(2005),395-405.

[13] Fadi Awawdeh, H.M. Jaradat, O. Alsayyed, "Solving system of DAEs by homotopy analysis method", Chaos, Solutions and Fractals 42 (2009) 1422-1427,Elsevier.

[14] H.Jafari, M.Zabihi and M.Saidy.Application of Homotopy perturbation method for solving Gas Dynamic Equation.Appl.Math.sci.,2:2008),2393-2396.

[15] J.H.He.Homotopy perturbation method for solving boundary value problems.Phys Lett A.350 (12)(2006),87-88.

[16] J.H.He, Comparison of homotopy perturbation method and homotopy analysis method, Appl.Math. Method, 42(2004) 759-766.

[17] SJ.Liao, Comparision between the homotopy analysis method and homotopy perturbation method, Appl.Math.Comput. 169 (2005) 1186-94. 